\title{
AN EXPANSION IN TERMS OF ASSOCIATED LEGENDRE FUNCTIONS
}

\author{
by T. M. MACROBERT
}

(Received 29th November, 1948)

$\S 1$. Introductory. If the right-hand side of the expansion

$$
\left(1-2 \mu h+h^{2}\right)^{-\frac{1}{2}}=\sum_{n=0}^{\infty} h^{n} P_{n}(\mu)
$$

is integrated $m$ times from 1 to $\mu$, it becomes

$$
\left(\mu^{2}-1\right)^{\frac{1}{2} m} \sum_{n=0}^{\infty} h^{n} P_{n}^{-m}(\mu) .
$$

In Hobson's treatise on Spherical and Ellipsoidal Harmonics, page 105, it is stated that the corresponding integration of the left-hand side gives rise to the function

$$
\frac{\Gamma\left(\frac{1}{2}-m\right)}{2^{m} \Gamma\left(\frac{1}{2}\right)}\left(1-2 \mu h+h^{2}\right)^{m-\frac{1}{2}} h^{-m},
$$

together with a rational expression which involves only powers of $h$. If, however, the integration is carried out step by step it is seen that, after the first step, the rational expression involves powers of $\mu$ also and that it is of the form

$$
\frac{\Gamma\left(\frac{1}{2}-m\right)}{2^{m} \Gamma\left(\frac{1}{2}\right)} h^{-m} \sum_{n=0}^{2 m-1}(-1)^{n 2 m-1} C_{n} h^{n} f_{n}^{m}(\mu),
$$

where, for $n=0,1,2, \ldots, m-1, f_{n}^{m}(\mu)$ is a polynomial in $\mu$ of degree $n$; and, for $n=m, m+1, \ldots, 2 m-1$,

$$
f_{n}^{m}(\mu)=f_{2 m-1-n}^{m}(\mu) \text {. }
$$

When $\mu=1, f_{n}^{m}(\mu)$ takes the value 1 .

These results will be established in $\S 3$, and it will be shown that the polynomials $f_{n}^{m}(\mu)$ can be expressed in terms of Associated Legendre Functions of the Second Kind. In $\S 2$ some formulae, old and new, on which the proof is based, are given.

§2. Associated Legendre Function Formulae. If $m$ is not a positive integer and if $h$ is sufficiently small, then *

$$
\left(1-2 \mu h+h^{2}\right)^{m-\frac{1}{2}}=\frac{\left(\mu^{2}-1\right)^{\frac{1}{2} m} \Gamma\left(\frac{1}{2}\right)}{2^{-m} \Gamma\left(\frac{1}{2}-m\right)} \sum_{n=0}^{\infty} h^{n} \frac{\Gamma(n-2 m+1)}{n !} P_{n-m}^{m}(\mu),
$$

where, if $n$ is zero or a positive interger and $m$ is not integral,

$$
\begin{aligned}
P_{n-m}^{m}(\mu)= & \frac{2^{n-m} \Gamma\left(n-m+\frac{1}{2}\right)}{\Gamma\left(\frac{1}{2}\right) \Gamma(n-2 m+1)}\left(\mu^{2}-1\right)^{-\frac{1}{2} m} \mu^{n} \\
& \quad \times F\left(-\frac{1}{2} n, \frac{1}{2}-\frac{1}{2} n ; \frac{1}{2}+m-n ; \mu^{-2}\right) .
\end{aligned}
$$

Now the right-hand side of (3) is equal to

$$
\frac{\sin 2 m \pi}{\pi \cos m \pi} \frac{\Gamma\left(\frac{1}{2}\right) \Gamma(2 m-n)}{2^{m-n} \Gamma\left(m-n+\frac{1}{2}\right)} \frac{\left(\mu^{2}-1\right)^{\frac{1}{2} m}}{\mu^{2 m-n}} F\left(\frac{2 m-n}{2}, \frac{2 m-n+1}{2} ; \frac{1}{\mu^{2}}\right) .
$$

*Quart.J. of Math. (Oxford) XIV. (1943), I, 2. 
Therefore, if $n$ is zero or a positive integer and $m$ is not integral,

The formula

$$
P_{n-m}^{m}(\mu)=\frac{2 \sin m \pi}{\pi} Q_{m-n-1}^{m}(\mu) .
$$

will also be required.

$$
P_{n}^{m}(\mu)=\frac{\Gamma(n+m+1)}{\Gamma(n-m+1)} P_{n}^{-m}(\mu)+2 \frac{\sin m \pi}{\pi} Q_{n}^{m}(\mu)
$$

§ 3. Proof of the Expansion. On replacing $n$ by $n-m$ in (5), that formula becomes

$$
\Gamma(n-2 m+1) P_{n-m}^{m}(\mu)=\Gamma(n+1) P_{n-m}^{-m}(\mu)+2 \frac{\sin m \pi}{\pi} \Gamma(n-2 m+1) Q_{n-m}^{m}(\mu) .
$$

Hence, if $m$ tends to a positive integral value and $n \geqq 2 m$,

$$
\Gamma(n-2 m+1) P_{n-m}^{m}(\mu) \rightarrow n ! P_{n-m}^{-m}(\mu),
$$

a well-known formula.

Again, from (6),

$$
\Gamma(n-2 m+1) P_{n-m}^{m}(\mu)=n ! P_{n-m}^{--m}(\mu)+(-1)^{n} \frac{2 \sin m \pi}{\sin 2 m \pi} \frac{1}{\Gamma(2 m-n)} Q_{n-m}^{m}(\mu),
$$

and therefore, when $m$ tends to a positive integral value and $n=m, m+1, \ldots, 2 m-1$,

$$
\Gamma(n-2 m+1) P_{n-m}^{m}(\mu) \rightarrow n ! P_{n-m}^{-m}(\mu)+(-1)^{m+n} \frac{1}{\Gamma(2 m-n)} Q_{n-m}^{m}(\mu) .
$$

Next, from (4),

$$
\Gamma(n-2 m+1) P_{n-m}^{m}(\mu)=(-1)^{n} \frac{2 \sin m \pi}{\sin 2 m \pi} \frac{1}{\Gamma(2 m-n)} Q_{m-n-1}^{m}(\mu),
$$

and therefore, when $m$ tends to a positive integral value and $n=01,2, \ldots, m-1$,

$$
\Gamma(n-2 m+1) P_{n-m}^{m}(\mu) \rightarrow(-1)^{m+n} \frac{1}{\Gamma(2 m-n)} Q_{m-n-1}^{m}(\mu) .
$$

Thus, from (9), (8) and (7), when $m$ tends to a positive integral value, (2) becomes

$$
\begin{aligned}
& \left(1-2 \mu h+h^{2}\right)^{m-\frac{1}{2}}=\frac{\left(\mu^{2}-1\right)^{\frac{1}{2} m} \Gamma\left(\frac{1}{2}\right)}{2^{-m} \Gamma\left(\frac{1}{2}-m\right)} \\
& \quad \times\left\{\begin{array}{c}
\sum_{n=0}^{m-1}(-1)^{m+n} h^{n} \frac{1}{n ! \Gamma(2 m-n)} Q_{m-n-1}^{m}(\mu) \\
2 m-1 \\
+\sum_{n=m}^{2 m-1}(-1)^{m+n} h^{n} \frac{1}{n ! \Gamma(2 m-n)} Q_{n-m}^{m}(\mu)+\sum_{n=m}^{\infty} h^{n} P_{n-m}^{-m}(\mu)
\end{array}\right\} .
\end{aligned}
$$

Thus, finally,

$$
\begin{aligned}
& \frac{\Gamma\left(\frac{1}{2}-m\right)}{2^{m} \Gamma\left(\frac{1}{2}\right)}\left(1-2 \mu h+h^{2}\right)^{m-\frac{1}{2}}=\left(\mu^{2}-1\right)^{\frac{1}{2} m} \sum_{n=0}^{\infty} h^{n+m} P_{n}^{-m}(\mu) \\
& \quad+\sum_{n=0}^{m-1}(-1)^{m+n} h^{n} \frac{\left(\mu^{2}-1\right)^{\frac{1}{2} m}}{n !(2 m-1-n) !} Q_{m-n-1}^{m}(\mu) \\
& \quad+\sum_{n=m}^{2 m-1}(-1)^{m+n} h^{n} \frac{\left(\mu^{2}-1\right)^{\frac{1}{2} m}}{(2 m-1-n) ! n !} Q_{n-m}^{m}(\mu) . \ldots
\end{aligned}
$$

On replacing $n$ by $2 m-\mathrm{I}-n$ in the last line it is seen that the coefficient of $h^{2 m-1-n}$ is equal to minus the coefficient of $h^{n}$ in the second last line. But

$$
f_{n}^{m}(\mu)=\frac{2^{m} \Gamma\left(\frac{1}{2}\right)}{\Gamma\left(\frac{1}{2}-m\right)} \frac{n !(2 m-1-n) !}{(2 m-1) !} \times \text { coefficient of }(-h)^{n}
$$



in these two lines. Therefore

$$
f_{2 m-1-n}^{m}(\mu)=f_{n}^{m}(\mu)
$$

where $n=0,1,2, \ldots, m-1$.

Again, if $n=0,1,2, \ldots, m-1$,

$$
\begin{aligned}
f_{n}^{m}(\mu)= & \frac{2^{m} \Gamma\left(\frac{1}{2}+m\right)}{\Gamma\left(\frac{1}{2}\right) \Gamma(2 m)} \frac{\left(\mu^{2}-1\right)^{m}}{\mu^{2 m-n}} \frac{\Gamma\left(\frac{1}{2}\right) \Gamma(2 m-n)}{2^{m-n} \Gamma\left(m-n+\frac{1}{2}\right)} \\
& \quad \times F\left(\frac{2 m-n}{2}, \frac{2 m-n+1}{2} ; m-n+\frac{1}{2} ; \frac{1}{\mu^{2}}\right) \\
= & \frac{2^{n} \Gamma\left(\frac{1}{2}+m\right) \Gamma(2 m-n)}{\Gamma(2 m) \Gamma\left(m-n+\frac{1}{2}\right)} \mu^{n} F\left(\begin{array}{c}
-\frac{1}{2} n, \frac{1}{2}-\frac{1}{2} n \\
m-n+\frac{1}{2}
\end{array} ; \frac{1}{\mu^{2}}\right) .
\end{aligned}
$$

From this it is clear that $f_{n}^{m}(\mu)$ is a polynomial in $\mu$ of degree $n$.

Lastly, let $\mu \rightarrow 1$ and apply Gauss's Theorem; then

$$
f_{n}^{m}(1)=\frac{2^{n} \Gamma\left(\frac{1}{2}+m\right) \Gamma(2 m-n)}{\Gamma(2 m) \Gamma\left(m-n+\frac{1}{2}\right)} \frac{\Gamma\left(m-n+\frac{1}{2}\right) \Gamma(m)}{\Gamma\left(m-\frac{1}{2} n+\frac{1}{2}\right) \Gamma\left(m-\frac{1}{2} n\right)}=1 .
$$

UnIVERsity of Glasgow 